\title{
Moments of Record Values and Characterizations of Marshall-Olkin Extended Distribution
}

\author{
G.G. Hamedani \\ Department of Mathematics, Statistics and Computer Science, Marquette University \\ Milwaukee, WI 53201-1881 \\ g.hamedani@mu.edu \\ Miroslav M. Ristić \\ Faculty of Sciences and Mathematics \\ University of Nǐ̌, Serbia \\ miristic@ptt.rs
}

Received 1 May 2014

Accepted 1 August 2015

\begin{abstract}
Moments of the $m^{\text {th }}$ upper record value $X_{U(m)}, m \geq 1$, and the joint moments of the $m^{\text {th }}$ and $n^{\text {th }}$ upper record values $X_{U(m)}$ and $X_{U(n)}, n>m \geq 1$, of a random variable $X$ with Marshall-Olkin Extended distribution are presented. Certain characterizations of this distribution based on hazard function and a simple relationship between two truncated moments are established.
\end{abstract}

Keywords: Moments of Upper Record Values, Truncated moments

Mathematics Subject Classification: 62E10; 60E05; 60E15

\section{Introduction}

Adding parameters to a well-defined model is a novel way of constructing flexible families of univariate and bivariate distributions. For a better understanding of this point, we refer the interested reader to Marshall and Olkin works. Marshall-Olkin Extended (MOE) distribution, introduced by Al-Jarallah et al. [1] has $p d f$ (probability density function) and $c d f$ (cumulative distribution function) given respectively by

$$
f(x)=f(x ; \alpha, \delta)=\frac{\alpha \delta k(x)[\alpha \bar{K}(x)]^{\delta-1}}{[1-\bar{\alpha} \bar{K}(x)]^{\delta+1}}, \quad x \in \mathbb{R}
$$

and 


$$
F(x)=F(x ; \alpha, \delta)=1-\left[\frac{\alpha \bar{K}(x)}{1-\bar{\alpha} \bar{K}(x)}\right]^{\delta}, \quad x \in \mathbb{R}
$$

where $k(x)=\frac{d}{d x} K(x)$ is the baseline $p d f, \bar{K}(x)=1-K(x)$ and $\alpha>0(\bar{\alpha}=1-\alpha), \delta>\mathbf{0}$ are parameters.

The $p d f$ of the $m^{\text {th }}$ upper record value, $X_{U(m)}$, of a random variable with $p d f(1.1)$ is given by

$$
f_{X_{U(m)}}(x)=\frac{\alpha^{\delta} \delta^{m}}{(m-1) !}\left(-\log \left(\frac{\alpha \bar{K}(x)}{1-\bar{\alpha} \bar{K}(x)}\right)\right)^{m-1} \frac{k(x)(\bar{K}(x))^{\delta-1}}{(1-\bar{\alpha} \bar{K}(x))^{\delta+1}}, \quad x \in \mathbb{R}
$$

On the other hand, the joint probability density function of the $m^{\text {th }}$ and $n^{\text {th }}$ upper record values $X_{U(m)}$ and $X_{U(n)}, n>m \geq 1$, of a random variable with $p d f(1.1)$ is given by

$$
\begin{aligned}
f_{X_{U(m)}, X_{U(n)}}(x, y) & =\frac{\alpha^{\delta} \delta^{n}}{(m-1) !(n-m-1) !}\left(-\log \frac{\alpha \bar{K}(x)}{1-\bar{\alpha} \bar{K}(x)}\right)^{m-1} \\
& \times\left(-\log \left(\frac{1-\bar{\alpha} \bar{K}(x)}{\bar{K}(x)} \cdot \frac{\bar{K}(y)}{1-\bar{\alpha} \bar{K}(y)}\right)\right)^{n-m-1} \\
& \times \frac{k(x) k(y)(\bar{K}(y))^{\delta-1}}{\bar{K}(x)(1-\bar{\alpha} \bar{K}(x))(1-\bar{\alpha} \bar{K}(y))^{\delta+1}}, x<y .
\end{aligned}
$$

The rest of the paper is organized as follows. In Section 2, we derive the moments of the $m^{\text {th }}$ upper record value $X_{U(m)}, m \geq 1$ and the joint moments of the $m^{\text {th }}$ and $n^{\text {th }}$ upper record values $X_{U(m)}$ and $X_{U(n)}, n>m \geq 1$. Then we present certain characterizations of MOE distribution in Section 3.

\section{Moments of Record values of the MOE family of distributions}

As we mentioned in the Introduction, we derive, in this Section, the moments of the $m^{\text {th }}$ upper record value $X_{U(m)}, m \geq 1$ and the joint moments of the $m^{\text {th }}$ and $n^{\text {th }}$ upper record values $X_{U(m)}$ and $X_{U(n)}, n>m \geq 1$ of a random variable $X$ which has $p d f$ given by (1.1). Let us first consider the single moments. The moments of the random variable $X_{U(m)}$ are given by the following two theorems.

Theorem 2.1. Let us suppose that a random variable $X$ has the pdf given by (1.1). Let us suppose that the inverse function of the survival function $\bar{K}(x)$ can be rewritten in power series form as $\bar{K}^{-1}(x)=\sum_{i=0}^{\infty} a_{i}\left(x-x_{0}\right)^{i}, 0<x<1, x_{0} \in[0,1]$. Let $\left\{c_{i}\right\}$ and $\left\{b_{k}\right\}$ be two sequences given by $c_{0}=a_{0}^{l}, b_{0}=1, c_{i}=\left(i a_{0}\right)^{-1} \sum_{j=1}^{i}(l j-i+j) a_{j} c_{i-j}$ and $b_{k}=k^{-1} \sum_{j=1}^{k}(i j-k+j) \bar{\alpha}^{j} b_{k-j}$. If $0<\alpha<2$, then the moments of the random variable $X_{U(m)}$ are given as

$$
E\left(X_{U(m)}^{l}\right)=\delta^{m} \sum_{i=0}^{\infty} c_{i} \sum_{j=0}^{i}\left(\begin{array}{l}
i \\
j
\end{array}\right)\left(-x_{0}\right)^{i-j} \sum_{k=0}^{\infty} b_{k} \sum_{r=0}^{k}\left(\begin{array}{l}
k \\
r
\end{array}\right)(-1)^{r}(\delta+j+r)^{-m}
$$


Proof. The $l^{\text {th }}$ moment of the random variable $X_{U(m)}$ is given by

$$
E\left(X_{U(m)}^{l}\right)=\frac{\alpha^{\delta} \delta^{m}}{(m-1) !} \int_{-\infty}^{\infty} x^{l}\left(-\log \frac{\alpha \bar{K}(x)}{1-\bar{\alpha} \bar{K}(x)}\right)^{m-1} \frac{k(x)(\bar{K}(x))^{\delta-1}}{(1-\bar{\alpha} \bar{K}(x))^{\delta+1}} d x
$$

Setting $t=-\log \left(\frac{\alpha \bar{K}(x)}{1-\bar{\alpha} \bar{K}(x)}\right)$, the above integral can be rewritten as

$$
E\left(X_{U(m)}^{l}\right)=\frac{\delta^{m}}{(m-1) !} \int_{0}^{\infty}\left(\bar{K}^{-1}\left(\frac{e^{-t}}{\alpha+\bar{\alpha} e^{-t}}\right)\right)^{l} t^{m-1} e^{-\delta t} d t
$$

Using the power series expansion of the inverse function $\bar{K}^{-1}(x)$ and equation (0.314) of [5], we obtain

$$
\left(\bar{K}^{-1}\left(\frac{e^{-t}}{\alpha+\bar{\alpha} e^{-t}}\right)\right)^{l}=\left(\sum_{i=0}^{\infty} a_{i}\left(\frac{e^{-t}}{\alpha+\bar{\alpha} e^{-t}}-x_{0}\right)^{i}\right)^{l}=\sum_{i=0}^{\infty} c_{i}\left(\frac{e^{-t}}{\alpha+\bar{\alpha} e^{-t}}-x_{0}\right)^{i} .
$$

Replacing the last equation into (2.1), the $l^{\text {th }}$ moment of the random variable $X_{U(m)}$ can be rewritten as

$$
E\left(X_{U(m)}^{l}\right)=\frac{\delta^{m}}{(m-1) !} \sum_{i=0}^{\infty} c_{i} \sum_{j=0}^{i}\left(\begin{array}{l}
i \\
j
\end{array}\right)\left(-x_{0}\right)^{i-j} \int_{0}^{\infty}\left(\alpha+\bar{\alpha} e^{-t}\right)^{-j} t^{m-1} e^{-(\delta+j) t} d t .
$$

Let us consider the function $\left(\alpha+\bar{\alpha} e^{-t}\right)^{-j}$. Using the fact that $0<\alpha<2$, equation (0.314) of [5] and the binomial expansion we arrive at

$$
\left(\alpha+\bar{\alpha} e^{-t}\right)^{-j}=\left(1-\bar{\alpha}\left(1-e^{-t}\right)\right)^{-j}=\left(\sum_{k=0}^{\infty} \bar{\alpha}^{k}\left(1-e^{-t}\right)^{k}\right)^{j}=\sum_{k=0}^{\infty} b_{k} \sum_{r=0}^{k}\left(\begin{array}{l}
k \\
r
\end{array}\right)(-1)^{r} e^{-r t} .
$$

Replacing the last equation into (2.2), we obtain

$$
\begin{aligned}
E\left(X_{U(m)}^{l}\right) & =\frac{\delta^{m}}{(m-1) !} \sum_{i=0}^{\infty} c_{i} \sum_{j=0}^{i}\left(\begin{array}{l}
i \\
j
\end{array}\right)\left(-x_{0}\right)^{i-j} \sum_{k=0}^{\infty} b_{k} \sum_{r=0}^{k}\left(\begin{array}{l}
k \\
r
\end{array}\right)(-1)^{r} \int_{0}^{\infty} t^{m-1} e^{-(\delta+j+r) t} d t \\
& =\delta^{m} \sum_{i=0}^{\infty} c_{i} \sum_{j=0}^{i}\left(\begin{array}{l}
i \\
j
\end{array}\right)\left(-x_{0}\right)^{i-j} \sum_{k=0}^{\infty} b_{k} \sum_{r=0}^{k}\left(\begin{array}{l}
k \\
r
\end{array}\right)(-1)^{r}(\delta+j+r)^{-m} .
\end{aligned}
$$

Theorem 2.2. Let us suppose that a random variable $X$ has pdf given by (1.1). Let us suppose that the inverse function of the survival function $\bar{K}(x)$ can be rewritten in power series form as $\bar{K}^{-1}(x)=\sum_{i=0}^{\infty} a_{i}\left(x-x_{0}\right)^{i}, 0<x<1, x_{0} \in[0,1]$. Let $\left\{c_{i}\right\}$ and $\left\{d_{k}\right\}$ are two sequences given by $c_{0}=a_{0}^{l}, d_{0}=1, c_{i}=\left(i a_{0}\right)^{-1} \sum_{j=1}^{i}(l j-i+j) a_{j} c_{i-j}$ and $d_{k}=k^{-1} \sum_{j=1}^{k}(i j-k+j)\left(1-\frac{1}{\alpha}\right)^{j} d_{k-j}$. If $\alpha>1 / 2$, then the moments of the random variable $X_{U(m)}$ are given as 


$$
E\left(X_{U(m)}^{l}\right)=\delta^{m} \sum_{i=0}^{\infty} \frac{c_{i}}{\alpha^{i}} \sum_{j=0}^{i}\left(\begin{array}{l}
i \\
j
\end{array}\right)\left(-x_{0}\right)^{i-j} \sum_{k=0}^{\infty} d_{k}(\delta+j+k)^{-m}
$$

Proof. The proof is similar to that of Theorem 2.1. The difference is that the series expansion of the function $\left(\alpha+\bar{\alpha} e^{-t}\right)^{-j}$ is now given by

$$
\left(\alpha+\bar{\alpha} e^{-t}\right)^{-j}=\alpha^{-j}\left(1-\left(1-\frac{1}{\alpha}\right) e^{-t}\right)^{-j}=\alpha^{-j}\left(\sum_{k=0}^{\infty}\left(1-\frac{1}{\alpha}\right)^{k} e^{-k t}\right)^{j}=\alpha^{-j} \sum_{k=0}^{\infty} d_{k} e^{-k t}
$$

Using this expansion we obtain the expression for the $l^{\text {th }}$ moment of the random variable $X_{U(m)}$.

Example 2.1. Let us suppose that a random variable $X$ has the Marshall-Olkin extended exponential distribution, i.e. let us suppose that the survival function is $\bar{K}(x)=e^{-x}, x \geq 0$. The first two moments of the $n^{\text {th }}$ upper record value have been considered in Jose et al. [9]. Here we will present formula for derivation of $l^{\text {th }}$ moments, $l \geq 1$. The inverse survival function is

$$
\bar{K}^{-1}(x)=-\log x=\sum_{i=1}^{\infty} \frac{(-1)^{i}}{i}(x-1)^{i} .
$$

Thus, we have that $x_{0}=1$ and the coefficients $a_{i}$ are given as $a_{i}=(-1)^{i} / i, i \geq 1$. For derivation of moments we need the recurrence formulas for coefficients $c_{i}$. We have that $c_{0}=c_{1}=c_{l-1}=0$, $c_{l}=(-1)^{l}$, and

$$
c_{l+i}=\frac{1}{i} \sum_{j=0}^{i}(l j-i+j) \frac{(-1)^{j}}{j+1} c_{l+i-j}, i \geq 1 .
$$

Then the expression for moments follows from the above two theorems.

Example 2.2. Let us suppose that a random variable $X$ has the Marshall-Olkin extended Pareto distribution. Parent survival function is given as $\bar{K}(x)=x^{-\gamma}, x \geq 1, \gamma>0$, and the corresponding inverse function is

$$
\bar{K}^{-1}(x)=(1-x)^{-1 / \gamma}=\sum_{i=0}^{\infty}(-1)^{-1 / \gamma}\left(\begin{array}{c}
-1 / \gamma \\
i
\end{array}\right)(x-1)^{i} .
$$

The coefficients $a_{i}^{\prime} s$ are given as $a_{i}=\left(\begin{array}{c}-1 / \gamma \\ i\end{array}\right), i \geq 1$, and the coefficients $c_{i}$ are given as $c_{0}=1$ and

$$
c_{i}=\frac{1}{i} \sum_{j=1}^{i}(l j-i+j)\left(\begin{array}{c}
-1 / \gamma \\
j
\end{array}\right) c_{i-j}, i \geq 1
$$

Now, we will derive the joint moments of the $m^{\text {th }}$ and $n^{\text {th }}$ upper record values $X_{U(m)}$ and $X_{U(n)}$, $n>m \geq 1$, of a random variable $X$ with Marshall-Olkin Extended distribution. These joint moments are given by the following two theorems.

Theorem 2.3. Let us suppose that a random variable $X$ has $p d f$ given by (1.1). Let us suppose that the inverse function of the survival function $\bar{K}(x)$ can be rewritten in power series form as 
$\bar{K}^{-1}(x)=\sum_{i=0}^{\infty} a_{i}\left(x-x_{0}\right)^{i}, 0<x<1, x_{0} \in[0,1]$. Let $\left\{c_{i}\right\}$ and $\left\{d_{k}\right\}$ be two sequences given by $c_{0}=$ $a_{0}^{l}, d_{0}=1, c_{i}=\left(i a_{0}\right)^{-1} \sum_{j=1}^{i}(l j-i+j) a_{j} c_{i-j}$ and $d_{k}=k^{-1} \sum_{j=1}^{k}(i j-k+j) \bar{\alpha}^{j} d_{k-j}$. If $0<\alpha<2$, then the joint moments of the random variables $X_{U(m)}$ and $X_{U(n)}, n>m \geq 1$, are given as

$$
\begin{aligned}
E\left(X_{U(m)}^{r} X_{U(n)}^{s}\right) & =\delta^{n} \sum_{i=0}^{\infty} \sum_{j=0}^{\infty} c_{i} c_{j} \sum_{k=0}^{i} \sum_{l=0}^{j}\left(\begin{array}{l}
i \\
k
\end{array}\right)\left(\begin{array}{l}
j \\
l
\end{array}\right)\left(-x_{0}\right)^{i+j-k-l} \\
& \times \sum_{p=0}^{\infty} \sum_{w=0}^{\infty} d_{p} d_{w} \sum_{q=0}^{p} \sum_{h=0}^{w}\left(\begin{array}{l}
p \\
q
\end{array}\right)\left(\begin{array}{l}
w \\
h
\end{array}\right)(-1)^{q+h}(\delta+l+h)^{-n+m} \\
& \times(k+l+\delta+q+h)^{-m} .
\end{aligned}
$$

Proof. The joint moment of the random variables $X_{U(m)}$ and $X_{U(n)}$ is given by

$$
\begin{aligned}
E\left(X_{U(m)}^{r} X_{U(n)}^{s}\right) & =\frac{\alpha^{\delta} \delta^{n}}{(m-1) !(n-m-1) !} \int_{-\infty}^{\infty} x^{r}\left(-\log \frac{\alpha \bar{K}(x)}{1-\bar{\alpha} \bar{K}(x)}\right)^{m-1} \\
& \times \frac{k(x) d x}{\bar{K}(x)(1-\bar{\alpha} \bar{K}(x))} \int_{x}^{\infty} y^{s} \frac{k(y)(\bar{K}(y))^{\delta-1}}{(1-\bar{\alpha}(y))^{\delta+1}} \\
& \times\left(-\log \left(\frac{1-\bar{\alpha} \bar{K}(x)}{\alpha \bar{K}(x)} \cdot \frac{\alpha \bar{K}(y)}{1-\bar{\alpha} \bar{K}(y)}\right)\right)^{n-m-1} d y
\end{aligned}
$$

Letting $u=-\log \left(\frac{\alpha \bar{K}(x)}{1-\bar{\alpha} \bar{K}(x)}\right)$ and

$$
v=-\log \left(\frac{1-\bar{\alpha} \bar{K}(x)}{\alpha \bar{K}(x)} \cdot \frac{\alpha \bar{K}(y)}{1-\bar{\alpha} \bar{K}(y)}\right)
$$

the above integral can be rewritten as

$$
\begin{aligned}
E\left(X_{U(m)}^{r} X_{U(n)}^{s}\right) & =\frac{\delta^{n}}{(m-1) !(n-m-1) !} \int_{0}^{\infty}\left(\bar{K}^{-1}\left(\frac{e^{-u}}{\alpha+\bar{\alpha} e^{-u}}\right)\right)^{r} u^{m-1} e^{-\delta u} d u \\
& \times \int_{0}^{\infty}\left(\bar{K}^{-1}\left(\frac{e^{-(u+v)}}{\alpha+\bar{\alpha} e^{-(u+v)}}\right)\right)^{s} v^{m-1} e^{-\delta v} d v
\end{aligned}
$$

Now, following the same techniques used in Theorem 2.1 we obtain the proof of theorem.

Theorem 2.4. Let us suppose that a random variable $X$ has $p d f$ given by (1.1). Let us suppose that the inverse function of the survival function $\bar{K}(x)$ can be rewritten in power series form as $\bar{K}^{-1}(x)=\sum_{i=0}^{\infty} a_{i}\left(x-x_{0}\right)^{i}, 0<x<1, x_{0} \in[0,1]$. Let $\left\{c_{i}\right\}$ and $\left\{d_{k}\right\}$ are two sequences given by $c_{0}=a_{0}^{l}, d_{0}=1, c_{i}=\left(i a_{0}\right)^{-1} \sum_{j=1}^{i}(l j-i+j) a_{j} c_{i-j}$ and $d_{k}=k^{-1} \sum_{j=1}^{k}(i j-k+j)\left(1-\frac{1}{\alpha}\right)^{j} d_{k-j}$. If $\alpha>1 / 2$, then the joint moments of the random variables $X_{U(m)}$ and $X_{U(n)}$ are given as 


$$
\begin{aligned}
E\left(X_{U(m)}^{r} X_{U(n)}^{s}\right) & =\delta^{n} \sum_{i=0}^{\infty} \sum_{j=0}^{\infty} c_{i} c_{j} \sum_{k=0}^{i} \sum_{l=0}^{j}\left(\begin{array}{l}
i \\
k
\end{array}\right)\left(\begin{array}{l}
j \\
l
\end{array}\right)\left(-x_{0}\right)^{i+j-k-l} \alpha^{-(k+l)} \\
& \times \sum_{p=0}^{\infty} \sum_{q=0}^{\infty} d_{p} d_{q}(\delta+l+q)^{-n+m}(k+l+\delta+p)^{-m}
\end{aligned}
$$

Proof. Proof is similar to the proofs of Theorems 2.2 and 2.3.

\section{Characterization Results}

In designing a stochastic model for a particular modeling problem, an investigator will be vitally interested to know if their model fits the requirements of a specific underlying probability distribution. To this end, the investigator will rely on the characterizations of the selected distribution. Generally speaking, the problem of characterizing a distribution is an important problem in various fields and has recently attracted the attention of many researchers. Consequently, various characterization results have been reported in the literature. These characterizations have been established in many different directions. In this section, we present characterizations of MOE distribution. These characterizations are based on: $(i)$ hazard function; (ii) a simple relationship between two truncated moments. We like to mention that the characterization (ii) which is expressed in terms of the ratio of truncated moments is stable in the sense of weak convergence. It also serves as a bridge between a first order differential equation and probability.

\subsection{Characterizations based on hazard function}

The following definition is stated here for the sake of completeness.

Definition 3.1. Let $F$ be an absolutely continuous distribution with the corresponding $p d f f$. The hazard function corresponding to $F$ is denoted by $h_{F}$ and is defined by

$$
h_{F}(y)=\frac{f(y)}{1-F(y)}, \quad y \in \operatorname{Supp} F
$$

where Supp $F$ is the support of $F$.

It is obvious that the hazard function of a twice differentiable function satisfies the first order differential equation

$$
\frac{h_{F}^{\prime}(y)}{h_{F}(y)}-h_{F}(y)=q(y),
$$

where $q(y)$ is an appropriate integrable function. Although this differential equation has an obvious form since 


$$
\frac{f^{\prime}(y)}{f(y)}=\frac{h_{F}^{\prime}(y)}{h_{F}(y)}-h_{F}(y),
$$

for many univariate continuous distributions (3.2) seems to be the only differential equation in terms of the hazard function. The goal of the characterization based on hazard function is to establish a differential equation in terms of hazard function, which has as simple form as possible and is not of the trivial form (3.2). For some general families of distributions this may not be possible.

Proposition 3.1. Let $X: \Omega \rightarrow \mathbb{R}$ be a continuous random variable. The random variable $X$ has $p d f$ (1.1) if and only if its hazard function $h_{F}(x)$ satisfies the differential equation

$$
h_{F}^{\prime}(x)-k^{\prime}(x)(k(x))^{-1} h_{F}(x)=\frac{\delta(k(x))^{2}[1-2 \bar{\alpha} \bar{K}(x)]}{\{\bar{K}(x)[1-\bar{\alpha} \bar{K}(x)]\}^{2}}, \quad x \in \mathbb{R} .
$$

Proof. If $X$ has $p d f(1.1)$, then clearly (3.3) holds. Now, if (3.3) holds, then

$$
(k(x))^{-1} h_{F}^{\prime}(x)-k^{\prime}(x)(k(x))^{-2} h_{F}(x)=\frac{\delta(k(x))[1-2 \bar{\alpha} \bar{K}(x)]}{\{\bar{K}(x)[1-\bar{\alpha} \bar{K}(x)]\}^{2}},
$$

or

$$
\frac{d}{d x}\left\{(k(x))^{-1} h_{F}(x)\right\}=\frac{d}{d x}\left\{\delta[\bar{K}(x)(1-\bar{\alpha} \bar{K}(x))]^{-1}\right\}
$$

or

$$
\frac{f(x)}{1-F(x)}=h_{F}(x)=\frac{\delta k(x)}{\bar{K}(x)(1-\bar{\alpha} \bar{K}(x))}=\frac{\delta k(x)}{\bar{K}(x)}+\frac{\delta \bar{\alpha} k(x)}{1-\bar{\alpha} \bar{K}(x)} .
$$

Integrating both sides of the above equation from $-\infty$ to $x$, we arrive at

$$
-\log (1-F(x))=-\delta \log (\bar{K}(x))+\delta \log (1-\bar{\alpha} \bar{K}(x))+\delta \log (\alpha)
$$

from which we have

$$
1-F(x)=(\alpha \bar{K}(x))^{\delta}(1-\bar{\alpha} \bar{K}(x))^{-\delta}
$$

\subsection{Characterizations based on two truncated moments}

In this subsection we present characterizations of MOE distribution in terms of a simple relationship between two truncated moments. We like to mention here the works of Glänzel [2,3], Glänzel et al. (1984), Glänzel and Hamedani [4] and Hamedani [6-8] in this direction. Our characterization results presented here will employ an interesting result due to Glänzel [2] (Theorem 3.1 below). 
The advantage of the characterizations given here is that, $c d f F$ need not have a closed form and are given in terms of an integral whose integrand depends on the solution of a first order differential equation, which can serve as a bridge between probability and differential equation.

Theorem 3.1. Let $(\Omega, \mathscr{F}, \mathbf{P})$ be a given probability space and let $H=[a, b]$ be an interval for some $a<b(a=-\infty, b=\infty$ might as well be allowed $)$. Let $X: \Omega \rightarrow H$ be a continuous random variable with the distribution function $F$ and let $g$ and $h$ be two real functions defined on $H$ such that

$$
\mathbf{E}[g(X) \mid X \geq x]=\mathbf{E}[h(X) \mid X \geq x] \eta(x), \quad x \in H,
$$

is defined with some real function $\eta$. Assume that $g, h \in C^{1}(H), \eta \in C^{2}(H)$ and $F$ is twice continuously differentiable and strictly monotone function on the set $H$. Finally, assume that the equation $h \eta=g$ has no real solution in the interior of $H$. Then $F$ is uniquely determined by the functions $g, h$ and $\eta$, particularly

$$
F(x)=\int_{a}^{x} C\left|\frac{\eta^{\prime}(u)}{\eta(u) h(u)-g(u)}\right| \exp (-s(u)) d u
$$

where the function $s$ is a solution of the differential equation $s^{\prime}=\frac{\eta^{\prime} h}{\eta h-g}$ and $C$ is a constant, chosen to make $\int_{H} d F=1$.

We like to mention that this kind of characterization based on the ratio of truncated moments is stable in the sense of weak convergence, in particular, let us assume that there is a sequence $\left\{X_{n}\right\}$ of random variables with distribution functions $\left\{F_{n}\right\}$ such that the functions $g_{n}, h_{n}$ and $\eta_{n}$ $(n \in \mathbb{N})$ satisfy the conditions of Theorem 3.1 and let $g_{n} \rightarrow g, h_{n} \rightarrow h$ for some continuously differentiable real functions $g$ and $h$. Let, finally, $X$ be a random variable with distribution $F$ . Under the condition that $g_{n}(X)$ and $h_{n}(X)$ are uniformly integrable and the family $\left\{F_{n}\right\}$ is relatively compact, the sequence $X_{n}$ converges to $X$ in distribution if and only if $\eta_{n}$ converges to $\eta$, where

$$
\eta(x)=\frac{E[g(X) \mid X \geq x]}{E[h(X) \mid X \geq x]}
$$

This stability theorem makes sure that the convergence of distribution functions is reflected by corresponding convergence of the functions $g, h$ and $\eta$, respectively. It guarantees, for instance, the 'convergence' of characterization of the Wald distribution to that of the Lévy-Smirnov distribution if $\alpha \rightarrow \infty$, as was pointed out in Glänzel and Hamedani [4].

A further consequence of the stability property of Theorem 3.1 is the application of this theorem to special tasks in statistical practice such as the estimation of the parameters of discrete distributions. For such purpose, the functions $g, h$ and, specially, $\eta$ should be as simple as possible. Since the function triplet is not uniquely determined it is often possible to choose $\eta$ as a linear function. Therefore, it is worth analyzing some special cases which helps to find new characterizations reflecting the relationship between individual continuous univariate distributions and appropriate in other areas of statistics. 
Remark 3.1. (a) In Theorem 3.1, the interval $H$ need not be closed since the condition is only on the interior of $H$. (b) Clearly, Theorem 3.1 can be stated in terms of two functions $g$ and $\eta$ by taking $h(x) \equiv 1$, which will reduce the condition given in Theorem 3.1 to $E[g(X) \mid X \geq x]=$ $\eta(x)$. However, adding an extra function will give a lot more flexibility, as far as its application is concerned.

Proposition 3.2. Let $X: \Omega \rightarrow \mathbb{R}$ be a continuous random variable and let $h(x)=[1-\bar{\alpha} \bar{K}(x)]^{\delta+1}$ and $g(x)=h(x) \bar{K}(x)$ for $x \in \mathbb{R}$. The $p d f$ of $X$ is (1.1) if and only if the function $\eta$ defined in Theorem 3.1 has the form

$$
\eta(x)=\frac{\delta}{\delta+1} \bar{K}(x), \quad x \in \mathbb{R} .
$$

Proof. Let $X$ have $p d f(1.1)$, then

$$
(1-F(x)) \mathbf{E}[h(X) \mid X \geq x]=[\alpha \bar{K}(x)]^{\delta}, \quad x \in \mathbb{R},
$$

and

$$
(1-F(x)) \mathbf{E}[g(X) \mid X \geq x]=\frac{\delta}{\alpha(\delta+1)}[\alpha \bar{K}(x)]^{\delta+1}, \quad x \in \mathbb{R}
$$

and finally

$$
\eta(x) h(x)-g(x)=-\frac{1}{\delta}[1-\bar{\alpha} \bar{K}(x)]^{\delta+1} \bar{K}(x)<0, \quad x \in \mathbb{R} .
$$

Conversely, if $\eta$ is given as above, then

$$
s^{\prime}(x)=\frac{\eta^{\prime}(x) h(x)}{\eta(x) h(x)-g(x)}=\frac{\delta f(x)}{\bar{K}(x)}, \quad x \in \mathbb{R}
$$

and hence

$$
s(x)=-\log \left([\bar{K}(x)]^{\delta}\right), \quad x \in \mathbb{R} .
$$

Now, in view of Theorem 3.1, $X$ has $p d f$ (1.1).

Corollary 3.1. Let $X: \Omega \rightarrow \mathbb{R}$ be a continuous random variable and let $h(x)$ be as in Proposition 3.2. The pdf of $X$ is (1.1) if and only if there exist functions $g$ and $\eta$ defined in Theorem 3.1 satisfying the differential equation

$$
\frac{\eta^{\prime}(x) h(x)}{\eta(x) h(x)-g(x)}=\frac{\delta f(x)}{\bar{K}(x)}, \quad x \in \mathbb{R}
$$


Remark 3.2. (a) The general solution of the differential equation in Corollary 3.1 is

$$
\eta(x)=[\bar{K}(x)]^{-\delta}\left[-\int \delta f(x)[\bar{K}(x)]^{\delta-1}[1-\bar{\alpha} \bar{K}(x)]^{-(\delta+1)} g(x) d x+D\right],
$$

for $x \in \mathbb{R}$, where $D$ is a constant. One set of appropriate functions is given in Proposition 3.2 with $D=0$.

(b) Clearly there are other triplets of functions $(h, g, \eta)$ satisfying the conditions of Theorem 3.1. We presented one such triplet in Proposition 3.2.

\section{Acknowledgements}

The authors would like to thank the referees for their valuable suggestions improving the presentation of the content of this work.

\section{References}

[1] Al-Jarallah, R.A., Ghitany, M.E. and Gupta, R.C., A proportional hazard Marshall-Olkin extended family of distributions and its application to Gompertz distribution, Commun. Statist. Theory-Methods, $\mathbf{4 3}$ (2014), 4428-4443.

[2] Glänzel, W., A characterization theorem based on truncated moments and its application to some distribution families, Mathematical Statistics and Probability Theory (Bad Tatzmannsdorf, 1986), Vol. B, Reidel, Dordrecht, 1987, 75-84.

[3] Glänzel, W., Some consequences of a characterization theorem based on truncated moments, Statistics, 21 (1990), 613-618.

[4] Glänzel, W. and Hamedani, G.G., Characterizations of univariate continuous distributions, Studia Sci. Math. Hungar., 37 (2001), 83-118.

[5] Gradshteyn, I.S., Ryzhik, I.M., Table of Integrals, Series, and Products, Academic Press, New York, 2000.

[6] Hamedani, G.G., Characterizations of univariate continuous distributions. II, Studia Sci. Math. Hungar., 39 (2002), 407-424.

[7] Hamedani, G.G., Characterizations of univariate continuous distributions. III, Studia Sci. Math. Hungar., 43 (2006), 361-385.

[8] Hamedani, G.G., Characterizations of continuous univariate distributions based on the truncated moments of functions of order statistics, Studia Sci. Math. Hungar., 47 (2010), 462-484.

[9] Jose, K.K., Krishna, E., Ristić, M.M., On record values and reliability properties of Marshall-Olkin extended exponential distribution, J. Stat. Theory and Appl., 13 (2014), 247-262. 